\title{
Dependency level of babies on the neonatal unit: a comparison of two different classification systems
}

\author{
C W Yoxall, R W I Cooke, N J Shaw, N V Subhedar, A M Weindling
}

\begin{abstract}
Background-Monitoring activity on the neonatal unit is important for planning service provision and as part of monitoring quality of care. The dependency level of the patients cared for must be taken into account as well as the number of patients. Two different systems for determining dependency level are in common use. Aim-To develop a system that would allow the accurate determination of dependency level for babies in our care using both the British Association for Perinatal Medicine and Neonatal Nurses Association definitions and the Northern Neonatal Network definitions and to perform a comparison between these two systems. Method-Forty details relating to current clinical status and treatment being given were recorded daily for every patient on two neonatal units over a 17 month period. These details were recorded in a computer database, and dependency levels were calculated for each patient day using both systems.
\end{abstract}

Results-A total of 21905 patient days were recorded for 1555 patients. There was good agreement between the two systems on what constituted the highest level of dependency, but overall comparability was poor, with the two systems assigning comparable dependency levels to only $76 \%$ of patient days.

Conclusions-There is limited comparability in dependency levels between these two widely used systems. There is a need for a standardisation of definitions to allow meaningful comparisons to be made between units.

(Arch Dis Child Fetal Neonatal Ed 2001;85:F173-F176)

Keywords: dependency; neonatal units; service provision; quality of care

To provide an appropriate level of clinical service on a particular neonatal unit, it is necessary to know what the demand for that service is. Small, sick babies consume more health care resources than larger, less unwell babies. It is therefore not enough to know only about the number of patients cared for. A measure of the dependency level of the patients cared for on the unit is also required.

Table 1 Classification of dependency level using the BAPM/NNA system

Level 1 intensive care (maximal intensive care)

1. Receiving assisted ventilation (including intermittent positive airway pressure, intermittent mandatory ventilation, and constant positive airway pressure) and in the first 24 hours after its withdrawal.

Of less than 27 weeks gestation for the first 48 hours after birth.

With a birth weight less than $1000 \mathrm{~g}$ in the first 48 hours after birth.

Requiring major emergency surgery for the preoperative period and postoperatively for 48 hours. On the day of death.

Being transported by a team including medical and nursing staff. Who are receiving peritoneal dialysis.

Who require exchange transfusion complicated by other disease processes.

With severe respiratory disease in the first 48 hours of life requiring fractional inspired oxygen concentration $\left(\mathrm{FIO}_{2}\right)$ of $>0.6$

10. With recurrent apnoea needing frequent intervention, for example over five stimulations in eight hours or resuscitation with With recurrent apnoea needing frequent intervention, for example over five stimula
intermittent positive pressure ventilation (IPPV) two or more times in 24 hours.

11. With significant requirements for circulatory support, for example inotropes, three or more infusions of colloid in 24 hours or infusions of prostaglandins.

Level 2 intensive care (high dependency intensive care)

1. Requiring total parenteral nutrition.

Having convulsions.

Being transported by a skilled neonatal nurse alone.

With arterial line or chest drain.

With respiratory disease in the first 48 hours of life needing $\mathrm{FiO}_{2}$ of 0.4 to 0.6 .

With recurrent apnoea requiring stimulation up to five times in an eight hour period or any resuscitation with IPPV.

Who require an exchange transfusion alone.

Who are more than 48 hours postoperative and require complex nursing procedures.

With a tracheostomy for the first two weeks.

Special care

Care Unit, Liverpoo

Women's Hospital,

Liverpool, UK

C W Yoxall

R W I Cooke

N J Shaw

N V Subhedar

A $M$ Weindling

Correspondence to:

Dr Yoxall, Neonatal Intensive

Care Unit, Liverpoo

Women's Hospital, Crown

Street, Liverpool L8 7SS,

UK

Bill.yoxall@

lwh-tr.nwest.nhs.uk

Requiring continuous mo

Receiving added oxygen.

With tracheostomy after the first two weeks.

Being given intravenous glucose and electrolyte solutions.

Being tube fed.

Had minor surgery in the past 24 hours.

Receiving terminal care, but not on the day of death.

Being barrier nursed.

Undergoing phototherapy.

10. Receiving special monitoring (e.g. frequent glucose or bilirubin estimations).

11. Needing constant supervision (e.g. for babies of mothers who are drug abusers)

12. Being treated with antibiotics.

Normal care

1. Receiving care from the mother or mother substitute with medical or neonatal nursing advice if needed. 
Table 2 Classification of dependency level using the NNN system

Category $A$

Infants requiring artificial respiratory support

Category B

Infants requiring $40 \%$ or more oxygen

Infants whose entire fluid intake is provided intravenously

Infants whose current weight is less than $1000 \mathrm{~g}$

Infants with a stoma, or a pleural, peritoneal, or urethral drain in place

Category $C$

Infants receiving less than $40 \%$ supplemental oxygen

Infants receiving some intravenous fluid

Infants who are at least partially tube fed

Infants who currently weigh between 1000 and $1750 \mathrm{~g}$

Infants who have had a fit or a sustained ( $>20 \mathrm{~s}$ ) apnoeic attack during the previous 24 hours

Category D

Bottle or breast fed infants weighing more than $1750 \mathrm{~g}$ admitted for observation only

NNN, Northern Neonatal Network.

Table 3 Datapoints collected to determine dependency level

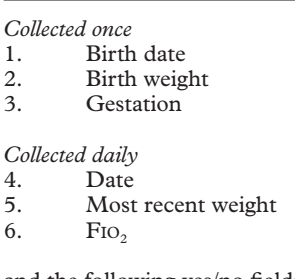

and the following yes/no fields

7. Ventilated in the past 24 hours?

8. CPAP in the last 24 hours?

9. Chest drain?

10. Arterial line?

11. Tracheostomy?

12. Receiving IV fluids other than parenteral nutrition?

13. Receiving parenteral nutrition?

14. Receiving tube feeds?

15. Receiving oral feeds?

Has a stoma?

Receiving inotropes?

Received volume expansion more than 3 times in past 24 hours?

Receiving prostaglandin infusion?

Undergoing peritoneal dialysis?

Received exchange transfusion in the past 24 hours?

Continuous monitoring?

Regular blood glucose measurements?

Receiving intravenous antibiotics?

Being barrier nursed?

Receiving phototherapy?

Apnoea requiring stimulation $>5$ times in 8 hours or IPPV $>2$ times in 24 hours?

Apnoea requiring stimulation $<5$ times in 8 hours or IPPV $\leqslant 2$ times in past 24 hours?

Having convulsions?

Experiencing drug withdrawal?

Receiving terminal care?

Day of death?

Pre-operative (major emergency surgery)?

$<24$ hours post minor surgery?

Major surgery in past 24 hours?

$>48$ hours postoperative but still requiring complex nursing?

Nurse transfer in past 24 hours?

Doctor and nurse transfer in past 24 hours?

$\mathrm{CPAP}$, continuous positive airway pressure; IV, intravenous; IPPV, intermittent positive pressure ventilation.
There are two dependency classifications in common use in the United Kingdom: that devised by The British Association for Perinatal Medicine and The Neonatal Nurses Association $^{3}$ (BAPM/NNA) (table 1) and that devised by the Northern Neonatal Network ${ }^{4}$ (NNN) (table 2). Both systems have four categories of dependency level, which appear to be broadly comparable. Both systems require knowledge of a number of items of information relating to a baby on any individual day in order to classify the dependency level of that patient day. Thirty two items are needed to accurately classify dependency level using the BAPM/NNA system, but only 11 are needed for the NNN system.

A recent study of retrospectively collected data has suggested that there is limited comparability between these two systems. ${ }^{2}$

The BAPM/NNA system is used on all of the neonatal units in the Mersey deanery. Dependency level is manually determined for each baby day by a member of the nursing staff on each unit. It has been our experience that there is a high level of inaccuracy in this system and high levels of disagreement between the people responsible for determining dependency level in these units.

The aims of this study were to develop a system that would allow the accurate determination of dependency level for babies in our care using both the BAPM/NNA and the NNN systems, and to perform a comparison between these two systems.

\section{Method}

Two different neonatal units provided information for the study. The neonatal unit at Liverpool Women's Hospital provides a regional intensive care service while the neonatal unit at University Hospital Aintree is a small special care baby unit which does not provide intensive care apart from a period of stabilisation before transfer to the regional centre. This provided data from a wide cross section of neonatal patients.

The data needed to determine dependency level by both systems were collected on each baby on the two neonatal units during each day of their admission and were entered into a prospectively maintained database constructed using standard software (Microsoft Access 95). The datapoints collected each day are shown (table 3), and the algorithm used to determine the dependency level are described (Appendix). These algorithms were incorporated into a database query to determine dependency level of each patient day using both systems. The study took place over a 17 month period, from 1 June 1998 to 31 October 1999. on a neonatal unit also allows comparisons to be made between different services and across time within the same unit. Because the duration of care at different dependency levels provides an indirect measure of the cost of care, recent studies have used dependency levels for economic evaluations of treatment. ${ }^{12}$ It is important that, for dependency information to be used in these ways, there must be agreement about the definitions used and those definitions must be applied in an accurate and consistent manner.

\section{Results}

A total of 1555 babies were cared for on the two units during the study period for a total of 21905 days. The median (range) gestation and birth weight were 35 (22-43) weeks and 2340 (420-7480) g respectively.

The overall division of these patient days into dependency levels using the two systems was broadly similar (table 4 ). The systems were 
Table 4 Overall dependency levels using the two systems

\begin{tabular}{|c|c|c|c|c|c|}
\hline \multicolumn{3}{|l|}{ NNN classification } & \multicolumn{3}{|l|}{ BAPM classification } \\
\hline Dependency level & $n$ & $\%$ & Dependency level & $n$ & $\%$ \\
\hline Category A & 3558 & 16 & Level 1 intensive care & 3900 & 18 \\
\hline Category B & 3530 & 16 & Level 2 intensive care & 2851 & 13 \\
\hline Category C & 12072 & 55 & Special care & 13141 & 60 \\
\hline Category D & 2745 & 13 & Normal care & 2013 & 9 \\
\hline
\end{tabular}

NNN, Northern Neonatal Network; BAPM, British Association for Perinatal Medicine; n, number of patient days.

Table 5 Comparability of dependency classification of patient days between the two systems

\begin{tabular}{lllll}
\hline & \multicolumn{2}{l}{ BAPM level } & & \\
\cline { 2 - 5 } NNN level & $\begin{array}{l}\text { Level 1 intensive } \\
\text { care }\end{array}$ & $\begin{array}{l}\text { Level 2 intensive } \\
\text { care }\end{array}$ & Special care & Normal care \\
\hline Category A & 3558 & 0 & 0 & 0 \\
Category B & 205 & 1286 & 1967 & 72 \\
Category C & 133 & 1558 & 10142 & 239 \\
Category D & 4 & 7 & 1032 & 1702 \\
\hline
\end{tabular}

NNN, Northern Neonatal Network; BAPM, British Association for Perinatal Medicine.

not, however, identical. Table 5 shows an analysis of the comparability of the two systems. There was agreement between the two systems in what constituted the highest level of dependency but comparability between the two systems was poor for the other categories, particularly the second level (Level 2 intensive care or Category B care). Overall the two systems assigned comparable dependency levels to only $76 \%$ of patient days.

\section{Discussion}

The computerised system appeared to work well. Every patient day was accounted for. The dependency level for each patient day was determined from the dataset recorded for each patient day using a predetermined algorithm. The dataset was a series of simple datapoints relating to the status of the baby and the care being given on that particular day. It did not contain any subjective interpretation of the baby's status by the staff providing the care. The algorithm always returns the same value for a given dataset. The system was therefore able to determine the dependency level of each patient day using both systems in an objective, reproducible manner.

The system can only be as accurate as the data entered, but each individual datapoint only requires knowledge of a single fact, which can be assessed objectively by those entering the data. No knowledge of the classification system is required by those entering the data. It is difficult to ensure complete objectivity in a system in which only the final classification is recorded, as detailed knowledge of the classification system is required by the individual and the eventual classification may be influenced by differences in individual perception, interpretation, or workload.

Because the details of the status of each baby and the treatment being given every day has been recorded, it would be possible to retrospectively determine the dependency level of the subjects using any other system developed.

Although the two systems are similar, they are not directly comparable. This precludes meaningful comparisons between units using the two different systems. Assessing dependency level allows the possibility to make useful comparisons in terms of performance and cost effectiveness. These comparisons can only be meaningful if a single system is agreed on. We must ensure that such a system can be collected accurately, completely, and objectively. The system must also meaningfully reflect the level of resources that the babies are consuming.

At present the NNN system has advantages over the BAPM/NNA system. It is simpler to collect because fewer data points need to be considered when determining dependency level. If the individual datapoints are collected, as in the approach described here, this reduces the size of the dataset significantly. If a "manual" system is used, then the simplicity of the NNN system suggests that it is more likely to be accurate. Previous analyses from the Northern Neonatal Network have reported better interobserver agreement for the NNN system than the BAPM/NNA system in both prospectively and retrospectively collected data. ${ }^{4}$ The NNN system also reflects the number of nurse hours received by a baby during each day $^{4}$ and is therefore validated as a measure of the resource requirements of the baby. No such validation has been performed for the BAPM/NNA system.

Both of the classification systems were developed almost a decade ago. There have been changes in neonatal care over that time and it seems timely to re-evaluate the methods we use to determine dependency level and to agree a national standard for data collection for the next decade.

\section{Appendix}

(1) Algorithm used to determine dependency level using BAPM/NNA definitions (numbers refer to datapoints in table 3)

If

either

7 or 8 or 17 or 18 or 19 or 20 or 27 or 32 or 33 or 35 or $38=$ "yes"

or

$((3=<27)$ and $(4-1=<3))$

or

$((2=<1000)$ and $(4-1=<3))$

or

$((6>60)$ and $(4-1=<3))$

or

$((21=$ "yes") and $(9$ or 11 or 13 or 16 or 24 or 29 or 34 or $36=$ "yes"))

Then

Else

[Dependency level] $=$ Level 1

If

either

9 or 10 or $(11$ and $(4-1>13))$ or 13 or 21 or 28 or 29 or 36 or $37=$ "yes"

$(6=($ between 40 and 60$))$ and $(4-1=<3)$

Then Else

[Dependency level] $=$ Level 2

If

either

12 or 14 or 22 or 23 or 24 or 25 or 26 or 30 or 31 or $34=$ "yes"

or

$6=>21$ 
or

Then

11 and $(4-1>13)$

Else

[Dependency level] $=$ Level 3

[Dependency level] $=$ Level 4 Ifend

Ifend

Ifend

2. Algorithm used to determine dependency level using the NNN definitions (numbers refer to datapoints in table 3)

If

7 or $8=$ "yes"

Then Else

$[$ Dependency level $]=$ Category $\mathrm{A}$

either

9 or 16 or $20=$ "yes"

or

$6=>40$

or

$(14=$ "no") and $(15=$ "no")

$5=<1000$

Then

[Dependency level] $=$ Category $\mathrm{B}$

Else
If

either

12 or 13 or 14 or 27 or 28 or $29=$ "yes" or

$6=($ between 22 and 40$)$

Then

$5=($ between $(1000$ and 1750)

[Dependency level] = Category C

Else Ifend

$[$ Dependency level $]=$ Category $\mathrm{D}$

Ifend

Ifend

Note-It is extremely unusual for babies in our care to have a urethral drain in situ and the algorithm makes no allowance for this intervention. Each day that a baby has a urethral drain should be assigned NNN category B. To incorporate this into the algorithm an extra data point (39. Urethral drain in situ) would need to be added to the data collected each day and the algorithm would need to be altered, changing 9 or 16 or $20=$ "yes" to 9 or 16 or 20 or $39=$ "yes".

1 Ainsworth SB, Beresford MW, Milligan DWA, et al. Pumactant and poractant alfa for treatment of respiratory distress syndrome in neonates born at 25-29 weeks gestation: a randomised trial. Lancet 2000;355:1387-92.

2 The ECSURF (Economic Evaluation of Surfactant) Collaborative Study Group. Limited comparability of classifications of levels of neonatal care in UK units. Arch Dis Child Fetal Neonatal Ed 1998;78:F179-84.

3 Report of working group of the British Association for Perinatal Medicine and Neonatal Nurses Association in categories of babies requiring neonatal care. Arch Dis Child 1992;67:868-9.

4 Northern Neonatal Network. Measuring neonatal nursing workload. Arch Dis Child 1993;68:539-43.

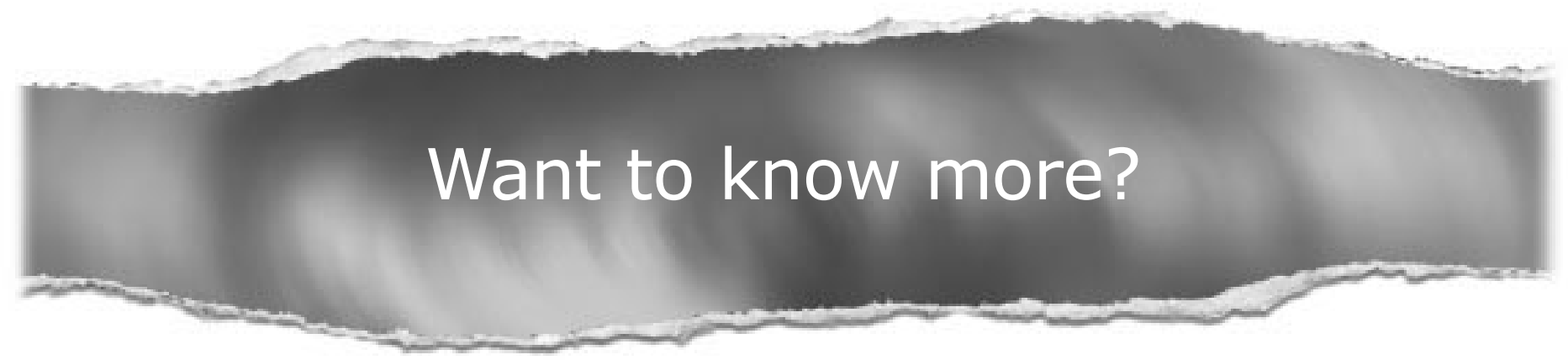

Data supplements

Limited space in printed journals means that interesting data and other material are often edited out of articles; however, limitless cyberspace means that we can include this information online.

Look out for additional tables, references, illustrations.

www.archdischild.com 